Associated Universities, Inc.

Upton, New York 11973

RHIC TeChnical Note No. 45

Internal Beam Dump System for RHIC

J. Claus, Horst Foelsche

$10 / 5 / 88$ 


\title{
INTERNAL BEAM DUMP SYSTEM FOR RHIC
}

\author{
J. Claus, H. Foelsche
}

\section{Introduction}

An internal beam dump for RHIC has been thought to be feasible, ${ }^{(1)}$ because the energy stored in the beam is relatively small, about $300 \mathrm{~kJ}$ per beam initially, and the expected secondary particle spray from the dump would not quench the superconducting magnets. The stored beam energy, if it can be spread over a sufficiently large dump volume within the constraints of the lattice, can be disposed of without resorting to special beam optics of an external channel. The effects of the secondary particle spray can be estimated by an analogy to the $\mathrm{CBA}$ extraction loss computations, and the problem is less serious than in the CBA case where the stored beam energy was to be 40 MJ. If one can maintain a reasonably similar geometric layout, the RHIC internal dump should therefore be expected to be safe for stored beam energies up to $300 \mathrm{~kJ}$, the initial design intensity goal.

Nevertheless, it may become necessary to extract the beam to an external dump in the future. Sooner or later the RHIC beam intensity will increase beyond the initial goals, and proton operation at ten times higher intensity has been mentioned. In that case the safe operating limits of the internal dump would be breached and the beam would have to be extracted to an external dump. If funding permits, a conservative approach favors extraction to start with. At the very least, an internal dump must be laid out in a configuration which can be upgraded to an extraction system later.

In this note we outline a limited initial scenario for an internal beam dump system. We do not undertake to describe an extraction system here, although we keep that option in view at all times. The internal dump is by no means a straightforward extrapolation from the CBA case. Even for the low initial design intensities, the beam cannot all be deposited in a fixed spot, but must be swept over a considerable area, lest it damage the dump material. Moreover, for heavy ions, the acceptance allowance required to accommodate intrabeam scattering is three times larger than what had been assumed for the protons in the CBA (about $6 \pi \mathrm{mm}$-mrad compared to $2 \pi \mathrm{mm}$-mrad, respectively). To copy an arrangement similar to the CBA layout, ${ }^{(2)}$ one has to enlarge several magnets between the kicker and the dump. Otherwise the larger beam envelope, on its way to the dump, will not fit into the aperture.

It would seem that, instead of copying the CBA layout, one could squeeze the kicker and an internal dump into one of the free spaces near an intersection region, or into a crossover region itself. Several of these alternatives are examined below, but found to be less desirable, or unacceptable. Either the internal dumps would be too close to superconducting components, and later extraction is precluded, or the beam dump and/or 
the associated secondary and tertiary protection collimators, and a beam extraction channel later, would claim an entire crossing region. Thus we conclude with a scenario, which geometrically resembles the CBA layout, and preserves the affected crossover region for experiments or machine physics research.

\section{Problems and Remedies}

The task is to eject a normally very small, potentially damaging beam from a relatively large "dump aperture", the phase space which the beam is permitted to occupy. In an emergency, when one detects an excursion toward the boundary of this dump aperture, one may not be in a position to control precisely where the beam is located. Therefore the entire permissible phase space must be transferred onto the dump. The response time of the system will be about $50 \mu \mathrm{sec}$ or less, to maintain control over the events in all conceivable cases of accidents or beam instabilities, and the beam will be aborted in a single turn $(-13 \mu \mathrm{sec})$. The same system will be used for routine disposal of beam before refilling the rings.

First, we must address the question of the survival of the dump under the impact of the beam. In disposing of the $300 \mathrm{~kJ}$ beam on an internal dump we must limit stresses on the dump material under worst case conditions, while accepting, without modification, the beam optics prevailing in the lattice. The beam is smallest immediately after having been accelerated to full energy, with a normalized emittance of $10 \pi \mathrm{mm}$-mrad at 2.5 standard deviations. Assuming, for simplicity, a joint Gaussian distribution of the beam density in the transverse plane, the peak density at the beam center is what would be obtained by distributing the beam uniformly over an "effective area" $2 \pi \sigma_{x} \sigma_{y}$. For the heaviest ions at the maximum energy (gold at $\beta \gamma=108$ ), depending on the chosen location, this effective area is about $2 \mathrm{~mm}^{2}$. This is the worst case, and for routine beam disposal one could consider decelerating the beam before aborting at lower energy, with a larger beam spot. There is no time for that in an emergency, and it is not enough in any event. Deposition in a single spot would overstress the dump material and would produce a meltdown. Beam cascade computations show that for small gold beams the maximum specific energy deposition occurs at the front face of the dump. For a small beam of heavy ions with a high charge the specific ionization at the front of the dump is higher than that of the developing cascade of low $Z$ secondary particles further downstream (in strong contrast to the situation for incident protons or light ions, or for very large beams, where the maximum develops further inside the material). Without further reference to cascade computations one obtains the result that one single bunch (of the 57 which make up a total of $300 \mathrm{~kJ}$.stored beam energy) will create a temperature impulse, resulting in temporary three-dimensional bulk stresses approaching or exceeding the yield stress of most absorber materials. The solution to this problem involves the choice of a material which has a low expansion coefficient, low modulus of elasticity, and high heat capacity, or very high strength, over a temperature range of 
several hundred degrees centigrade, and it may involve configurations employing twodimensional lamination stacks to relieve three-dimensional stress. The high strength alloy $\mathrm{Ti}-6 \mathrm{Al}-4 \mathrm{~V}$ would be a suitable candidate, providing adequate safety margin for a single bunch. Nevertheless, it is evident that one cannot deposit all 57 bunches in the same spot, and the beam must be spread over a much larger area. This can be accomplished by a twofold approach: the fast kicker current waveform can be designed to retain an oscillatory component around the maximum deflection amplitude (say in the vertical direction), while a slower "sweeper" moves the beam spot sideways. Figure 1 illustrates the idea. The pattern illustrated in this figure, although spread over about 60 $\mathrm{mm}^{2}$, would entail some overlapping of bunches, with very localized intensity enhancements of more than a factor of 2 over the maximum of a single isolated bunch. We conclude from all this that the initial design intensity goal for RHIC is not far from the limits of the dump tolerance. When the beam intensity increases beyond the initial design specification, and if the beam sweeping system cannot be enlarged further, then the beam must be extracted. Preliminary cascade simulations by Stevens ${ }^{(3)}$ confirm these conclusions.

Turning next to the problem of protecting superconducting magnets from secondary beam spray, one must consider the fact that very low specific energy depositions, on the order of millijoules/gram, can quench the superconducting magnets. Here one needs detailed simulations of the secondary particle cascades in the magnet lattice to obtain some guidance for the choice of layouts. For the case of RHIC, initial calculations look encouraging, provided that whatever magnets may be immediately behind the primary dump can be normal warm ones. A simple analogy to the CBA extraction concept can be used to obtain some reassurance on this point. We can assume with good confidence, and initial cascade computations ${ }^{(3)}$ confirm, that for the initial RHIC design intensities, the spray from beam buried far from the tangential face of the dump can be sufficiently supressed. While the kicker rises from zero to full field, however, a small fraction of the beam interacts in the tangential surface layer of the dump, and here is where most of the troublesome secondaries originate. This "surface loss fraction" (L) of the beam is proportional to some "effective thickness" of the surface layer (s), and inversely proportional to the rate of rise of the deflection [i.e. inversely proportional to the kicked beam displacement $(k)$, and proportional to the "effective rise time" ( $t)]$, viz.

$$
L=\frac{s}{k} \frac{t}{T} \text {, }
$$

where the rise time ( $t$ ) is assumed to be smaller than the beam revolution time (T). Initial cascade simulations indicate that at the highest energy the effective emitting surface layer is less than $1 \mathrm{~cm}$ thick for appropriate materials. For example, for a kick amplitude of $3 \mathrm{~cm}$, a rise time of $1 \mu \mathrm{sec}$, and a revolution period of $12.5 \mu \mathrm{sec}$, the effective loss 
fraction would then be less than $3 \%$. This fraction of $300 \mathrm{~kJ}$ amounts to a smaller source of secondaries than the beam loss on the CBA extraction septum $(0.04 \%$ of $40 \mathrm{MJ})$, which had been shown to be safe ${ }^{(4)}$ in the CBA configuration, and for RHIC the safe enthalpy reserve of the superconducting magnets may well be larger. Therefore, this estimate is not discouraging. The rise time of the kicker is the only variable that is readily available to reduce the surface loss. The problem can be avoided altogether if some rf buckets are left empty and the kicker rises during this gap in the beam. In that case the "surface loss fraction" is zero.

In the following discussion of aperture constraints we need to refer to various kinds of apertures which characterize the machine. By "lattice acceptance" we mean the geometric acceptance of the machine as defined only by its vacuum chamber openings and maximum beta values. It depends on the operating conditions, changing proportionally with the smallest $\beta^{*}$ in any of the insertions up to an upper limit, from about $6 \pi \mathrm{mm}$-mrad to $17 \pi \mathrm{mm}$-mrad when $\beta^{*}$ changes from $2 \mathrm{~m}-6 \mathrm{~m}$. Particles moving outside the lattice acceptance will hit the wall of the vacuum chamber within a very few revolutions. The "dynamic acceptance" represents the boundary of stable single particle motion, particles beyond this boundary will eventually be lost although that loss will take quite a few revolutions if they start close to it. The dynamic acceptance varies with the operating conditions, correction state, betatron wave numbers, momentum deviation, etc. and may be either larger or smaller than the lattice acceptance. It is zero by definition if the machine is unstable. Intrabeam scattering will, in the course of time, drive particles from within the dynamic acceptance across its boundary in its attempt to establish a truly Gaussian distribution in $6 \mathrm{D}$ phase space. A dynamic acceptance $\geq 140 \pi / \gamma$ $\mathrm{mm}$-mrad $(\gamma 28)$ seems to be adequate for a 10 hour luminosity lifetime for gold in RHIC. The "dump acceptance" and the "dump" aperture are concepts intended for the purpose of beam disposal in an emergency. If appropriate instrumentation detects that particles move across the dump acceptance boundary at too great a rate or, better, at a rate that increases too fast, the beam is dumped, because it is judged that the motion has become unstable. There are, of course, many other reasons why an emergency beam disposal might be triggered, e.g., excessive displacements of the closed orbit, coherent dipole motion with increasing amplitude, loss of r.f. power, detection of a magnet quench, etc. It is clear that the dump acceptance boundary must lie everywhere within the lattice acceptance at such a distance from it that all particles within it will reach the dump when the beam disposal process is triggered. Yet must it be large enough to allow normal operation to proceed unimpeded. The lattice acceptance is determined by the apertures of the high beta quadrupoles $Q_{2}$ and $Q_{3}$ under nearly all operating conditions. The dump acceptance might be defined by properly dimensioned collimating targets in their immediate vicinities. In the scenario outlined below we are adopting a dump. aperture phase space area of $6 \pi \mathrm{mm}$-mrad. 
Some fraction of the beam passing off-center through the kicker while its field is rising will be deflected outside the dump aperture, though not yet enough to be fully intercepted by the beam dump. In general such partially deflected beam, which we call the "whipping tail", cannot make it around the machine again, if the undeflected beam has been allowed to roam in a major fraction of the geometric lattice acceptance to begin with. This subject has already been treated rigorously in Reference (2) for the extraction of continuous beams from the CBA. Figure 2 illustrates the situation for the favored layout described further below. In the normalized phase space coordinate system employed in this figure, the beam dump is located at the edge of a dump aperture ellipse of area $6 \pi \mathrm{mm}$-mrad, represented here by a circle of "unit" radius. The minimum acceptance ellipse which, in an emergency, would contain all possible whipping tails depends on the betatron phase advance between kicker and dump, and would be, as a minimum, at least twice the phase space which the beam was permitted to occupy before disposal, and is in fact four times larger in the most favorable of the possible cases discussed below. If there is insufficient geometric aperture reserve in the lattice, the whipping tail must be intercepted on secondary beam dumps other than the primary one, before it enters the lattice again. There must be several of these secondary beam dumps, all of which are likely to radiate secondaries from their tangential surface layer, and therefore have tertiary collimators in their wake to protect downstream superconductors. An arrangement of this type would consume the entire space of an experimental intersection region. A theoretical, but unacceptable alternative would be to restrict the permissible dump aperture to a suitable fraction of the geometric acceptance (about one quarter in our most favored case, or about $2.5 \pi \mathrm{mm}$-mrad) so that all possible whipping tails fit into the lattice as well. These considerations remain unchanged for an extracted beam. Again, a gap in the beam during the kicker rise will simplify the problem. In that case there is no whipping tail, the experimental area remains free of secondary dumps, and the dump aperture can be larger.

The aperture constraints imposed by the beam dump or extraction process apply principally in the plane in which the beam abort process takes place. If the kicker acts in the vertical plane, the restrictions in the horizontal and longitudinal phase space may be less severe.

What needs to be done in the horizontal plane will depend on the effects of the high energy quasielastic and small angle neutral and negative secondaries, which emerge predominantly from the tangential surface layer of the dumps. These are the components of the spray which are not sufficiently dispersed by the quadrupoles near the dump and which do not leave the beam envelope until they encounter the first bending magnet downstream. Neutrals and some negative secondaries will hit the vacuum chamber of the first dipoles downstream, and the quasielastics will separate out even further on. The detailed collimator configurations cannot be settled without cascade computations more 
extensive than have been performed so far. Once again, the source of all these problems virtually disappears, if there is a gap in the beam during the kicker rise, so that the tangential surface of the dump is not exposed to beam.

It can be seen that there are several compelling reasons to favor operation of RHIC with a gap of several empty if buckets. Allowing the kicker to rise to full field during this gap, will eliminate both the spray from the surface layer of the dump and the need for secondary dumps and tertiary collimators to intercept the whipping tail. The small angle and quasielastic spray is virtually eliminated. These statements are equally true for an extraction system, where the spray would originate on the thin current sheet of the extraction septum magnet, and the whipping tail has to be dealt with as well.

It is important to keep in mind, however, that introducing gaps of certain duration in the two counterrotating beams will reduce the interaction time for the experimenter by twice that amount on each turn, except in the two areas where the gaps can be made to cross at the same time. In view of the required size of the kicker equipment it is quite unrealistic to expect the kicker to rise in the time between two regular bunches (initially 110-220 nsec, perhaps even less later). A time gap of one microsecond in the beam will cost four of the six experimenters roughly one sixth of their time-averaged luminosity (one twelfth for the other two). The alternative is to withdraw one of the six intersections from the experimental program altogether and to dedicate it exclusively to the beam dump system and the array of secondary dumps and tertiary collimators. In that case the remaining five experimenters may enjoy the full luminosity.

\section{Choices}

In Figure 3 we illustrate four seemingly realistic configurations for an internal beam dump system. In order to narrow down the choices among these alternatives, it is useful to ask the following three questions about each of them:

1. Is a gap in the beam required to protect magnets immediately following the dump? As a general rule we will require that if there are quadrupoles immediately behind primary or secondary beam dumps, they shall be normal warm magnets. Furthermore we will require that the nearest bending magnets be at least $40 \mathrm{~m}$ away to protect them from small angle neutral spray. If we cannot meet these two requirements, we will require a gap in the beam to avoid all secondary particle spray from the surface layers of the dumps.

2. Is beam extraction possible in the future? Beam extraction is to be accomplished by replacing the internal dump by a septum magnet string, and an external beam line, for which there must be sufficient clear space.

3. Can the insertion, where the dump system is located, be preserved for the experimental program?

The Table 1 below summarizes the answers to these questions for all four of the possible configurations. Figure 3 illustrates the layouts. 
TABLE 1. INTERNAL BEAM DUMP OPTIONS

\begin{tabular}{|c|c|c|c|c|}
\hline & $\begin{array}{l}\text { Location } \\
\text { Kicker } \rightarrow \text { Dump } \\
\quad(\text { See Fig. } 3 \text { ) }\end{array}$ & $\begin{array}{l}\text { Beam Gap } \\
\text { Required? }\end{array}$ & $\begin{array}{c}\text { Future } \\
\text { Extraction } \\
\text { Possible? }\end{array}$ & $\begin{array}{c}\text { Experimental } \\
\text { Intersection } \\
\text { Preserved? }\end{array}$ \\
\hline (1) & $\mathrm{Q} 3 \rightarrow \mathrm{Q} 4$ & Always! & No & Yes \\
\hline (2) & Q3 $\rightarrow$ Crossover & Probably & Yes & No \\
\hline (3) & $\mathrm{Q} 4 \rightarrow \mathrm{Q} 3$ & Always! & No & Yes \\
\hline (4) & $\mathrm{Q} 8 \rightarrow \mathrm{Q} 4$ & or $\left\{\begin{array}{l}\text { Yes } \\
\text { No }\end{array}\right.$ & $\begin{array}{l}\text { Yes } \\
\text { Yes }\end{array}$ & $\begin{array}{l}\text { Yes } \\
\text { No }\end{array}$ \\
\hline
\end{tabular}

Eventually the beam may have to be extracted from the machine. There are several reasons why it is necessary to keep this option open. First, the internal dump is highly stressed, and one's ability to spread the energy deposition over a large volume is limited by the constraints of the lattice. At some higher intensity one must take the beam outside for further enlargement before disposal in the dump. The second reason is that for much higher intensities even the relatively small remaining secondary spray, from deep within the dump, will become large enough to quench some superconducting elements. Also one may note that an extraction septum conductor can be thinner than the effective surface layer of a dump, and therefore may present a relatively weaker source of secondary spray. Although the internal dump appears safe for the initial operation of RHIC, it would be wrong to foreclose the option of extracting the beam at a later date. Therefore, the options (1) and (3) are unacceptable.

For the two remaining options (2) and (4), if extraction should be required in the future, the kicker system would remain the same and the primary internal dump would be replaced by an extraction septum magnet string, followed by an external dump further downstream.

Option (2) would surrender a crossover region to the beam dump for all time. The entire intersection region would then be optimized for this purpose, to the exclusion of all other uses. One of the intersection dipoles $(\mathrm{BC} 1)$ would be removed, and the other (BC2) shortened, or become a normal warm magnet. The available free space would then be filled with the primary dump and auxiliary protective collimators. In fact, the dump would be located at the intersection point, and one will design a special high-beta focus for this area to help enlarge the beam on the dump. If there were no gap in the beam, one might catch the whipping tail in this area too, but there may not be enough room then to collimate spray from these secondary dumps. Therefore, having given up the experimental area, one would probably have to maintain a gap in the beam anyway.

The last solution, option (4), preserves the experimental area, as long as a beam gap is employed. It is the only one that permits extraction of the beam at a later time while 
keeping the experimental crossover available. The nearest superconductors are about $35 \mathrm{~m}$ from the beam dump, rather than very nearby, and therefore this is technically the safest solution as well. Although this is the favored configuration in all respects, it is by no means easy to implement. The apertures of the quadrupoles Q6 and Q4 must be enlarged, and the dipoles BS1 and BS2 must be lowered a little, to permit the kicked beam to pass through them in this particular region. The beam dump would be located downstream of Q4. In spite of the required aperture enlargements, however, this is the layout that should be adopted.

One may continue to keep both options (2) and (4) in mind until final decisions are made, and on closer examination one or the other layout may encounter insurmountable technical difficulties. In the meantime it is useful to lay out an internal dump scenario based on the most favorable option (4).

\section{Internal Beam Dump Scenario}

To summarize, the internal beam dump remains a possible approach for the initial heavy ion intensity of RHIC, and for an initial proton intensity of $10^{11}$ protons per bunch, i.e for up to $300 \mathrm{~kJ}$ stored beam energy, provided an extraction option is kept open for higher performance parameters later. The favored option (4) is adopted for the purpose of further planning.

The beam dump system can be installed in the inner arcs of the 6 o'clock insertion, where it was to be located for CBA, or in the undeveloped insertion region at 10 o'clock. In these areas the lattice parameters favor dumping in the vertical plane, so that the impact of the aperture restrictions in the horizontal and in the longitudinal dimension are minimized. The kicker system will be designed to handle a dump aperture of $6 \pi \mathrm{mm}$ mrad.

Figures 4 and 5 illustrate the envelopes containing a dump aperture phase space area of $6 \pi \mathrm{mm}$-mrad, with and without the action of the kicker and the sweeper, for the two cases where the beta function at the crossover is $6 \mathrm{~m}$ and $3.2 \mathrm{~m}$, respectively. The kicker may be located on both sides of the quadrupole Q8. The figures also show, as a shaded area surrounding the deflected vertical envelope, the additional room required for the residual oscillation, which is imposed on the kicker field. It is difficult to accommodate the horizontal sweeper magnet. The best that can be done is a position is near the quadrupole Q5, perhaps with an assist from an additional short component near Q6. In order to gain sufficient horizontal lever arm with respect to the sweeper, the primary beam dump will be placed about $7 \mathrm{~m}$ downstream of $\mathrm{Q} 4$, a somewhat less than ideal position from the point of view of the required vertical kick. The kicker deflection strength will be about $1.2 \mathrm{Tm}$ with a full magnetic aperture of about $7 \times 7 \mathrm{~cm}^{2}$, and the sweeper will have about the same strength. The kicker rise time will be about $1 \mu \mathrm{sec}$, while the sweeper will rise in about $13 \mu \mathrm{sec}$. The apertures of the quadrupoles Q6 and Q4 must have an inner bore tube radius of $63 \mathrm{~mm}$, and the bending magnets BS1 and 
BS2 will be lowered by $10-15 \mathrm{~mm}$. It may also be advantageous to widen the aperture of Q5, and to use a warm bore vacuum chamber there, to ease the transition between the cryogenic quadrupole and the sweeper magnet in this very confined region. These modifications are required only in the inner arcs near the affected intersection.

In the early operation of RHIC, a gap of $1.1 \mu \mathrm{sec}$ duration will be left in the beam, and the machine is commissioned up to the initial design intensity in this manner. It is likely to be advantageous to maintain the gap for a considerable time in the life of the machine, if one can safely handle higher intensities in this fashion as well, and also for the case of beam extraction later. Only the primary beam dump should be built initially, together with auxiliary collimators which intercept the now much reduced secondary spray from deep within the dump.

Preliminary beam cascade computations by Stevens ${ }^{(3)}$ confirm that the internal dump scenario outlined here should be viable with sufficient safety margin, for the beam intensities initially envisioned for RHIC. The ultimate confirmation has to be empirical. The inherent limitation of the internal beam dump is reached when, given a maximum effort to spread the beam over the largest possible volume, the particle cascade density gives rise to internal stresses which damage the dump. Then one needs to carry the beam outside the machine for further enlargement before disposal.

The simulations of secondary particle energy deposition in the superconducting magnets are also encouraging. Here it would be useful to commit higher computing power to increase the statistical significance of the Monte Carlo computations. It is necessary to keep in mind, however, that these cascade simulations do not promise an inherent accuracy of better than a factor of three in some instances. If they should show that the internal dump system will operate with what is judged to be too small a safety margin, then a conservative plan would favor an extraction system at the outset.

We have not discussed costs in this note. One now needs to confirm the technical concepts before making another cost estimate. The largest single element of the beam dump is the kicker system. The kicker will be designed for a rise time of about $1 \mu s e c$. This is three times faster than had been assumed in the original RHIC design report, and the kicker is stronger than for the CBA. The sweeper magnet, about the same strength as the kicker, with a much slower rise time of $13 \mu \mathrm{sec}$, has to be added as an additional protective element. One may say with fair certainty that the cost of the kicker and sweeper systems will be twice what had been originally extrapolated from the CBA case. For an extraction line, one would add the cost of a septum magnet string and its power supplies, and also add additional beam enlargement systems.

\section{References}

(1) RHIC Conceptual Design Report, BNL Report \#51932, May 1986.

(2) J. Claus \& H. Foelsche, Extraction for ISABELLE, IEEE Trans. Nucl. Science, NS28 (1981), 3065. (BNL-29233). 
(3) A. Stevens, RHIC Internal Beam Dump; Preliminary Conceptual Design. Internal Report RHIC-43 (1988).

(4) A. Stevens, Radiation Heating from Beam Loss on ISABELLE Ejection Septa, IEEE Trans. Nucl. Science, NS-28 (1981), 2924. (BNL-29055). 

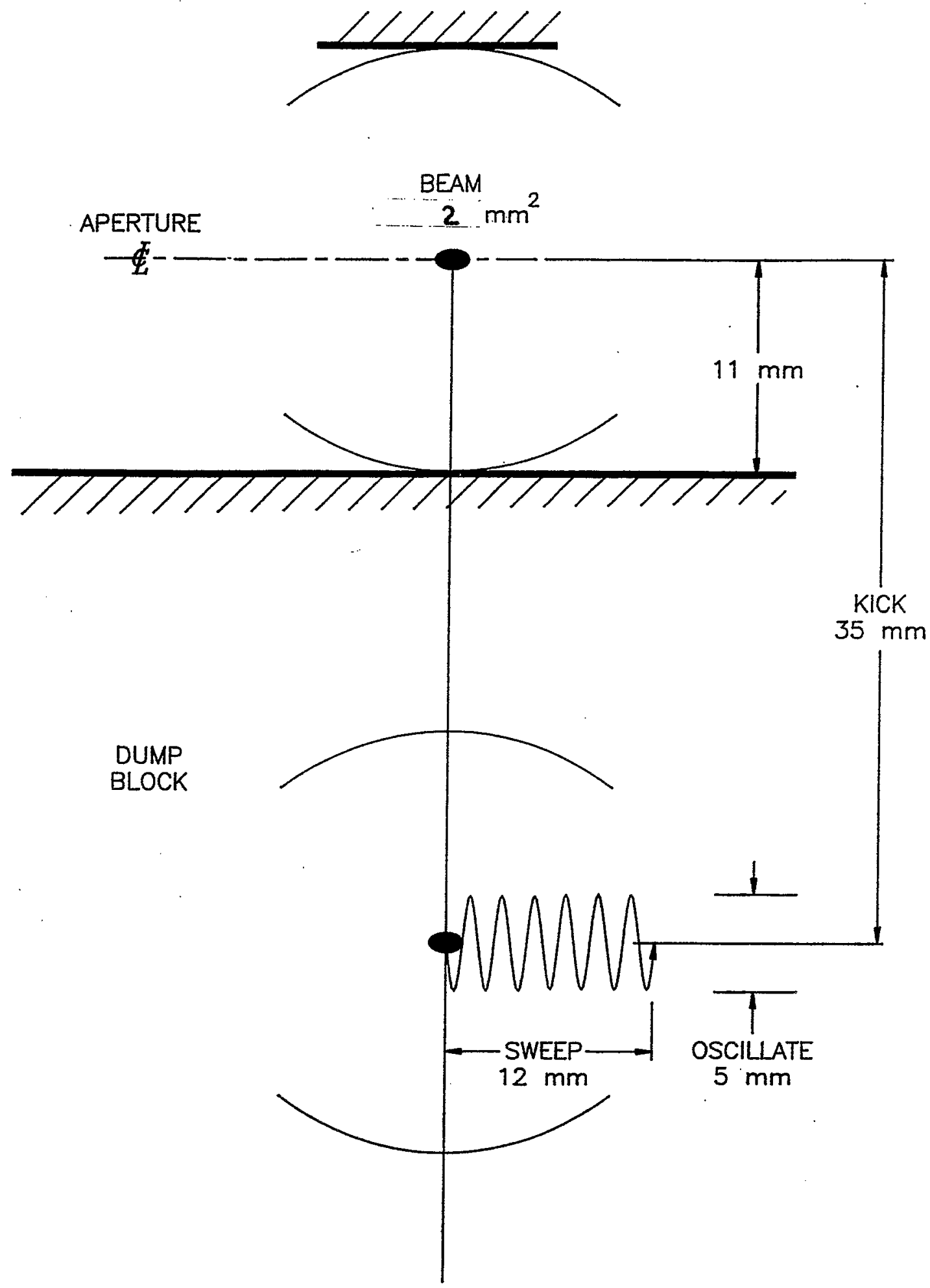

Fig. 1. Beam Dump Schematic (viewed head-on). Beam is located within the dump aperture, before being deflected into the dump block by a fast kicker magnet. A residual oscillation in the kicker magnet current, and the action of a horizontal sweeper magnet, spread this beam over an appropriate dump volume, to avoid excessive stresses. 


$$
\beta^{*}=6 \mathrm{~m}
$$

KICKERS: NEAR Q8

BEAM DUMP: $7 \mathrm{~m}$ BEYOND Q4

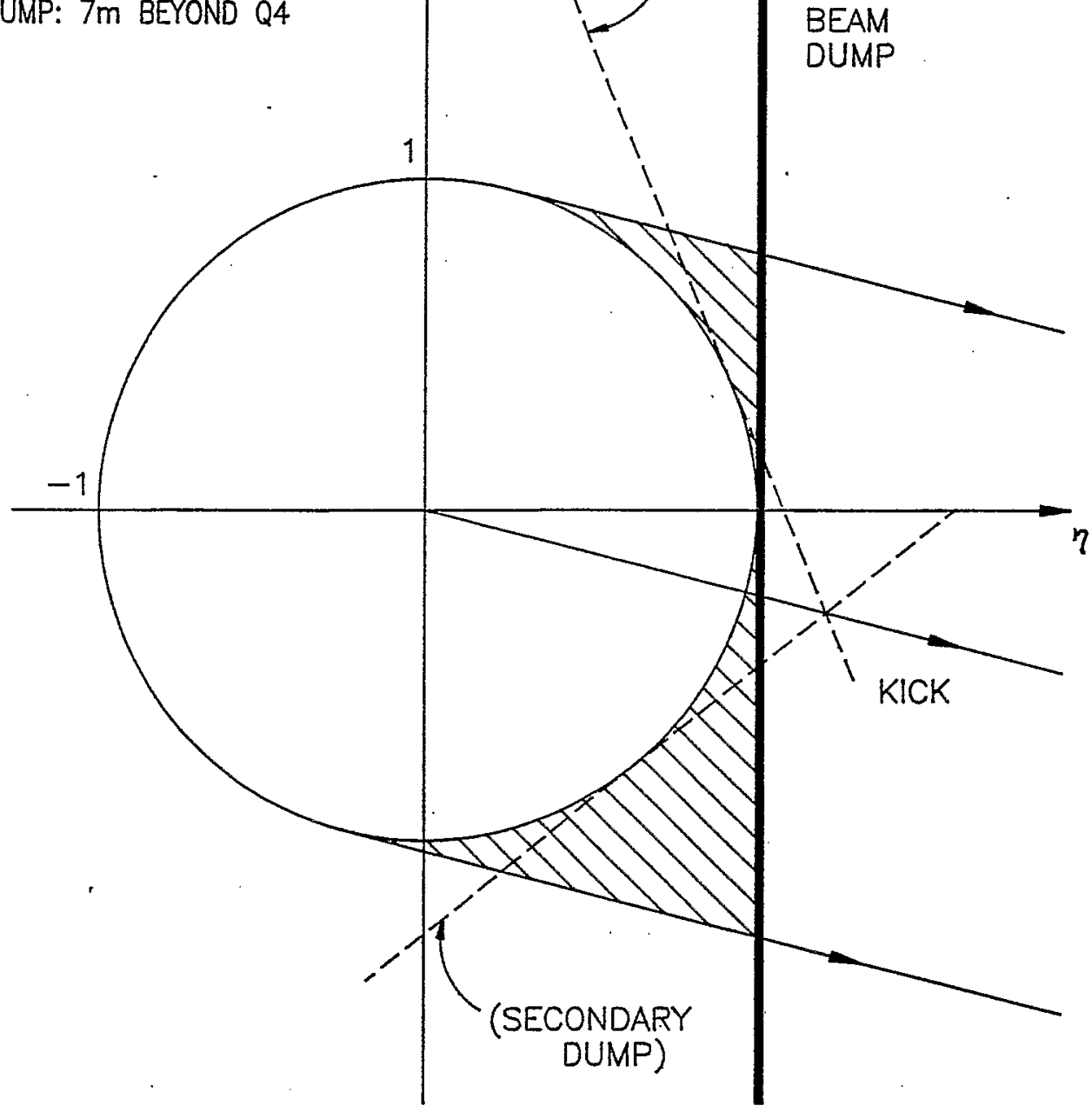

Fig. 2. Vertical phase space at beam dump, in normalized coordinates, where dump aperture ellipse is represented by a unit circle. While kicker rises to full field, some partially deflected, off-center beam could be found outside dump aperture, without being intercepted by dump block: the "whipping tail" (shaded area). Secondary beam dumps are needed to intercept these particles, unless a gap in the beam during the kicker rise avoids "whipping tail" altogether. 
INTERNAL DUMP OPTIONS

INNER ARC

OUTER

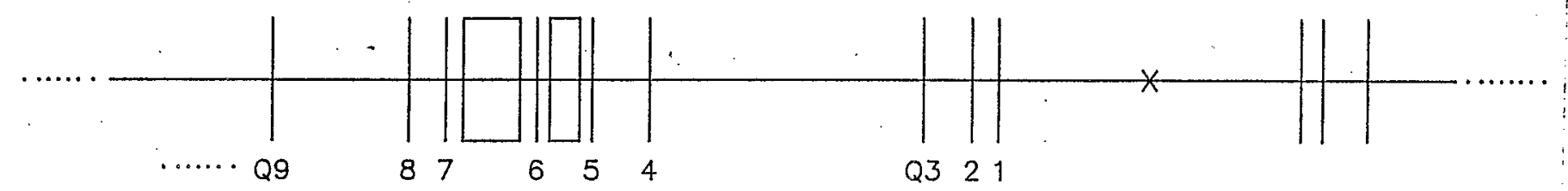

1. $Q 3 \rightarrow Q 4$

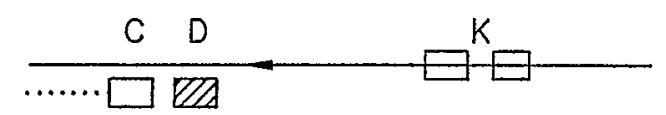

2. $Q 3-X$

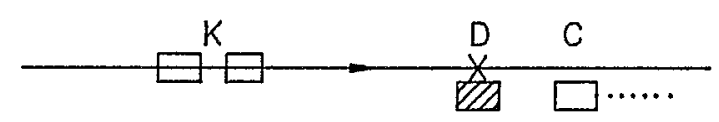

3. $Q 4-Q 3$

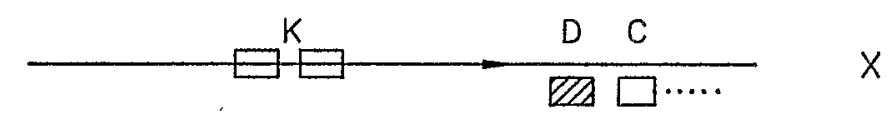

4. $Q 8-Q 4$

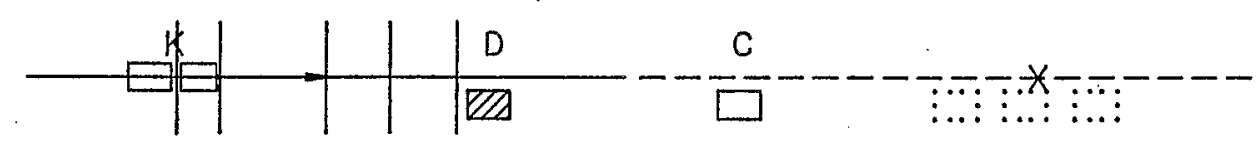

K - KICKER

D - DUMP

C - COLLIMATOR

$\because \cdots:-$ SECONDARY DUMPS \& COLLIMATORS

Fig. 3. Internal Dump Configurations for RHIC. Options (1) and (3) cannot be expanded to an extraction system later. Option (2) uses a crossover region. Option (4) is preferred. 
Vertical Kickers

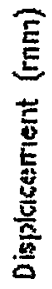

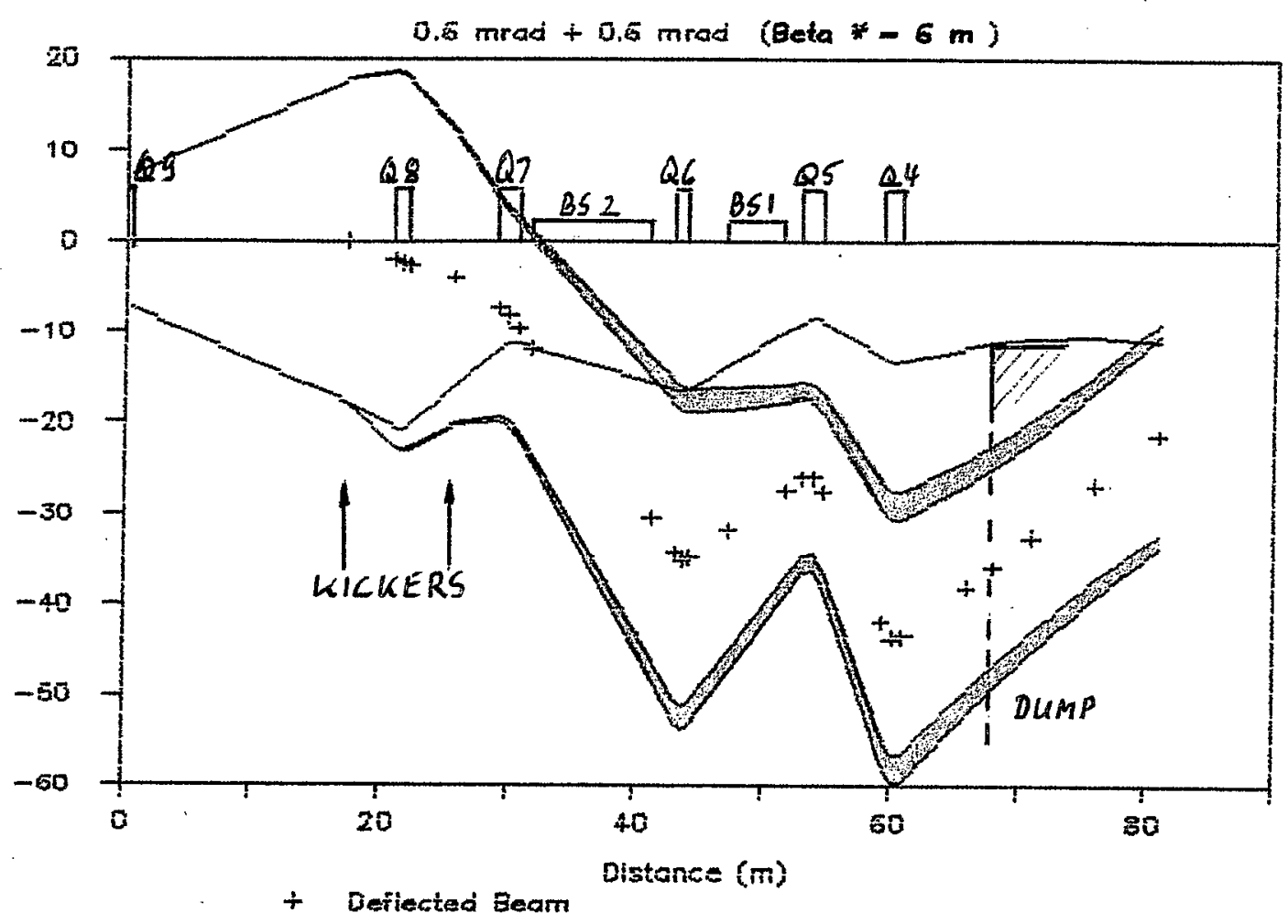

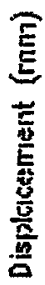

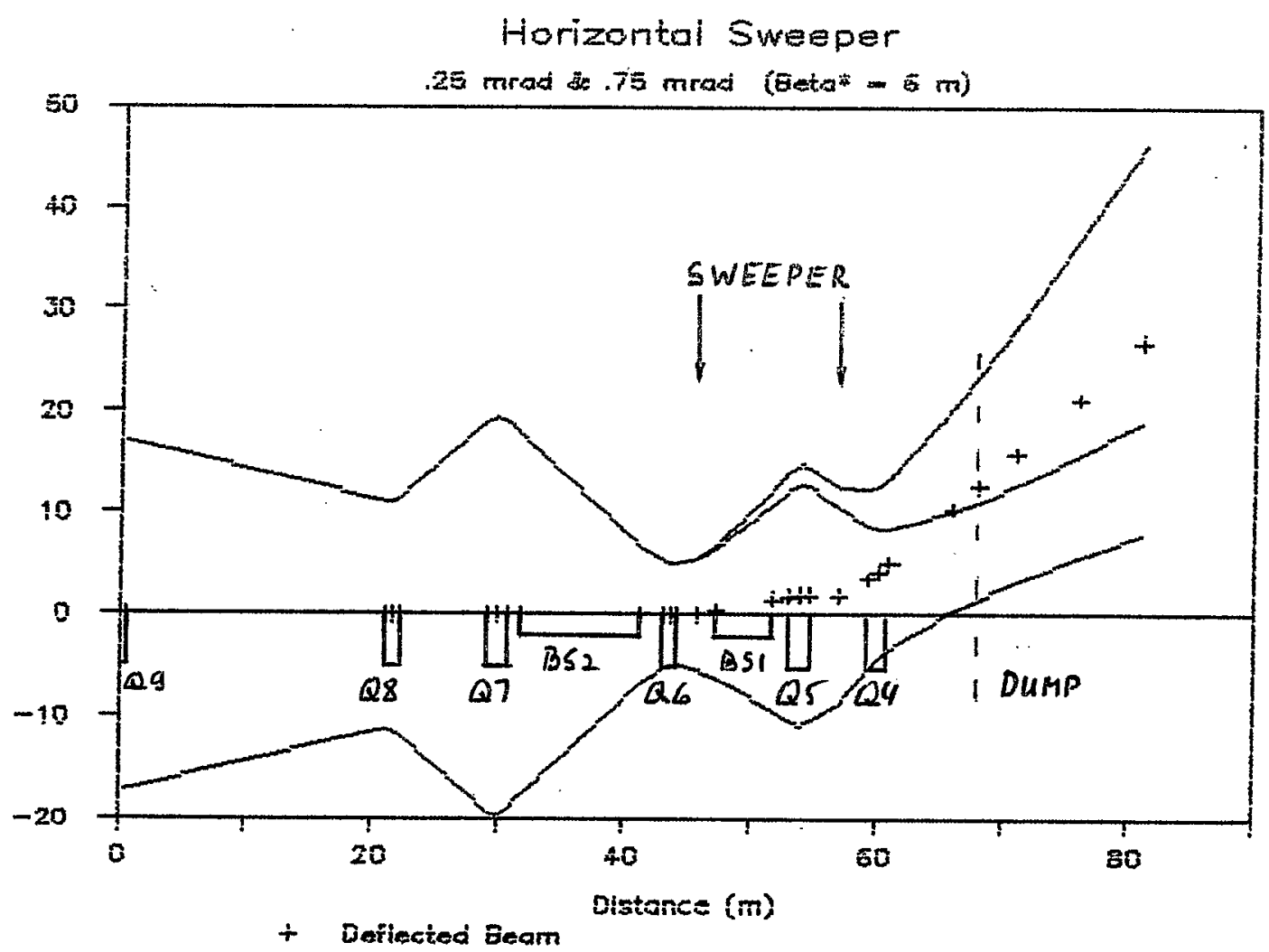

Fig. 4. Vertically and horizontally deflected acceptance envelopes between kicker and beam dump; including oscillating component (shaded) for spreading small beams; for dump aperture $=6 \pi \mathrm{mm}-\mathrm{mrad}, \beta^{*}=6 \mathrm{~m}$. 


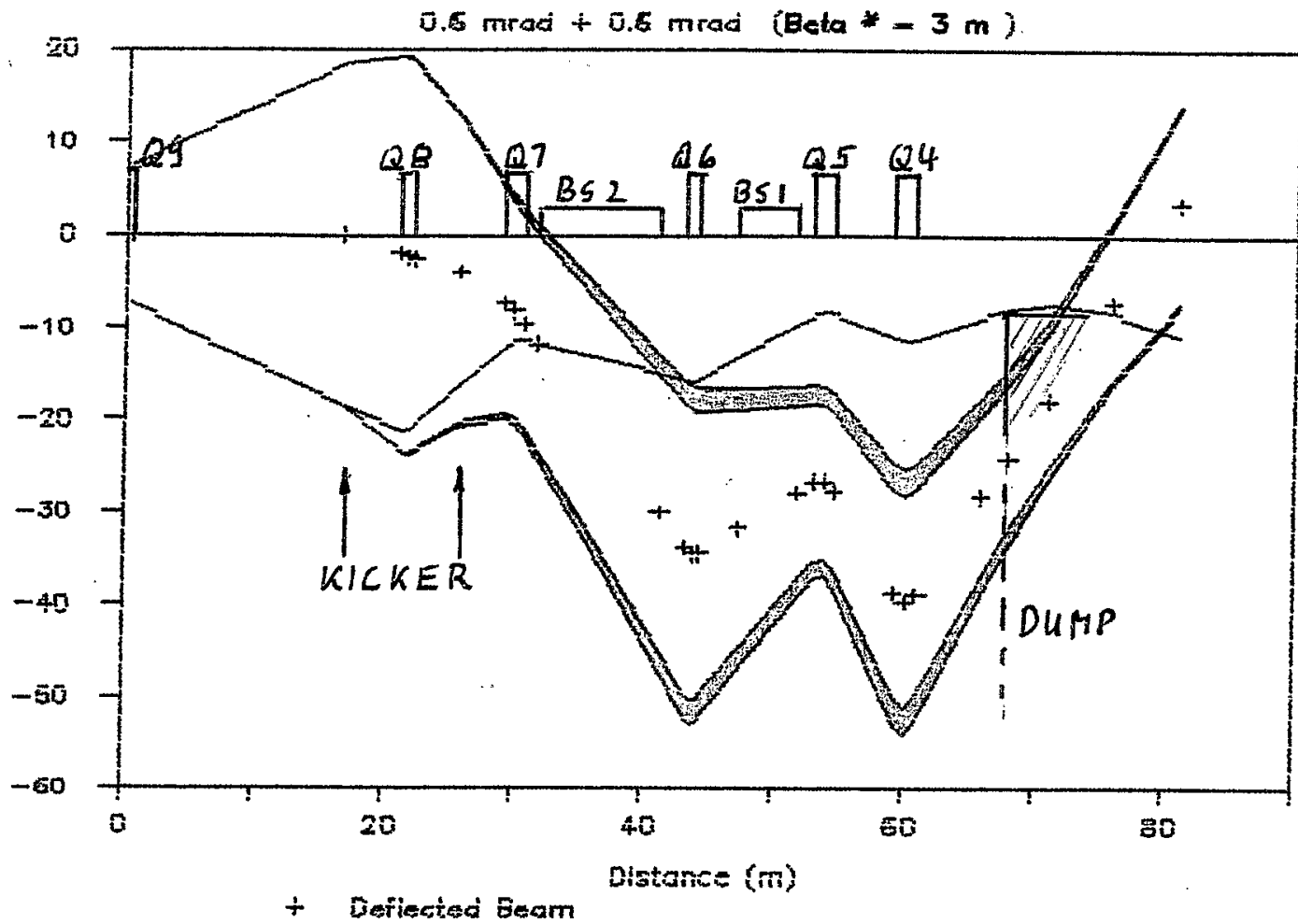

E
E
草
$\frac{5}{5}$
$\frac{5}{5}$
$\frac{5}{5}$

Horizontal Sweeper

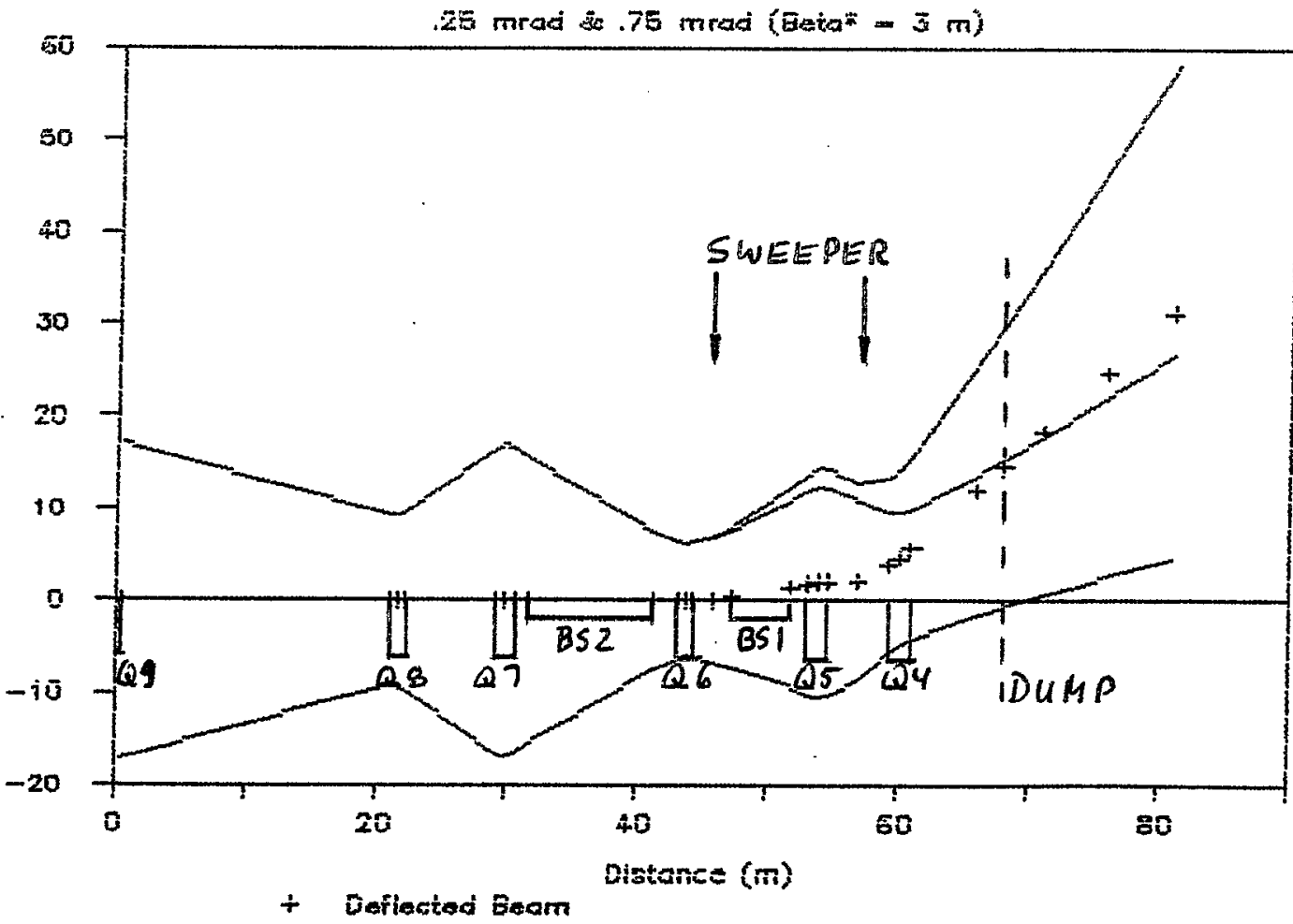

Fig. 5. Vertically and horizontally deflected acceptance envelopes between kicker and beam dump; including oscillating component (shaded) for spreading small beams; for dump aperture $=6 \pi \mathrm{mm}-\mathrm{mrad}, \beta^{*}=3.2 \mathrm{~m}$. 